\title{
LncRNA DSCAM-AS1 promotes non-small cell lung cancer progression via regulating miR-577/HMGB1 axis
}

\author{
Z. QIU*, X. X. PAN ${ }^{*}$, D.Y. YOU*
}

Department of Oncology, Thoracic, Head and Neck Surgery, Huangshi Central Hospital, Affiliated Hospital of Hubei Polytechnic University, Huangshi, Hubei, China

*Correspondence: kofpuh@163.com

"Contributed equally to this work.

Received August 26, 2019 / Accepted November 13, 2019

\begin{abstract}
Non-small cell lung cancer (NSCLC) is a major source of cancer mortality. Long non-coding RNA DSCAM-AS1 has been certified to be involved in the pathogenesis of NSCLC. This study aimed to further investigate the potential mechanism of DSCAM-AS1 in NSCLC progression. The expressions of DSCAM-AS1, miR-577, and high mobility group box 1 (HMGB1) were detected by quantitative real-time polymerase chain reaction (qRT-PCR) or western blot assay. Cell viability was assessed by Cell Counting Kit-8 (CCK-8) assay. Flow cytometry assay was conducted to monitor cell apoptosis. Cell migration and invasion were measured by transwell assay. Wnt/ $\beta$-catenin pathway-related factors were detected by western blot assay. The relationship between DSCAM-AS1, miR-577, and HMGB1 was validated by bioinformatics analysis and dual-luciferase reporter assay. The xenograft mouse model was established to analyze tumor growth in vivo. DSCAM-AS1 and HMGB1 were upregulated, while miR-577 was downregulated in NSCLC tissues and cells. DSCAM-AS1 promoted cell proliferation, migration and invasion, and restrained cell apoptosis in NSCLC cells. Overexpression of HMGB1 reversed the effects of DSCAM-AS1 depletion on the progression of NSCLC. DSCAM-AS1 modulated HMGB1 expression by sponging miR-577. DSCAM-AS1 regulated the Wnt/ $\beta$-catenin pathway by regulating miR-577 and HMGB1. DSCAM-AS1 knockdown blocked the tumor growth in vivo. In conclusion, DSCAM-AS1 facilitated NSCLC progression by regulating the HMGB1-mediated Wnt/ $\beta$-catenin pathway, providing a promising therapeutic target for NSCLC.
\end{abstract}

Key words: DSCAM-AS1, miR-577, HMGB1, Wnt/ß-catenin pathway, non-small cell lung cancer

Lung cancer is a common malignancy worldwide, accompanied by high morbidity and mortality [1]. Lung cancer is composed of $85 \%$ non-small cell lung cancer (NSCLC) and $15 \%$ small cell lung cancer (SCLC) [2]. The prognosis of patients with NSCLC is very poor, with a five-year survival rate of approximately $16 \%$ [3]. Therefore, it is of considerable significance to explore the underlying mechanisms of NSCLC in search of new therapeutic strategies.

Long non-coding RNAs (lncRNAs) are non-coding transcripts longer than 200 nucleotides (nt). Numerous studies have shown that lncRNAs exerted a regulatory role in different biological processes during tumorigenesis and development [4]. For instance, lncRNA HCG11 participated in cell proliferation, cycle arrest, and apoptosis in glioma via targeting miR-496 and elevating CPEB3 expression [5]. LncRNA NR2F2-AS1 facilitated NSCLC progression via cooperating with miR-320b/BMI1 axis [6]. Also, lncRNA XIST silencing hindered cell proliferation through sponging
miR-744 and enhancing RING1 axis expression in NSCLC [7]. Furthermore, recent researches have demonstrated that DSCAM-AS1 is involved in the regulation of several cancers, such as breast cancer [8]. In NSCLC, DSCAM-AS1 triggered cell migration and invasion via enhancing BCL11A expression [9]. Hence, we aimed to further investigate the mechanism of DSCAM-AS1 in NSCLC development.

MicroRNAs (miRNAs) are short non-coding RNAs composed of 18-25 nt. Increasing evidence has revealed that miRNAs modulate post-transcriptional levels through binding to target mRNAs [10]. Previous researches have elucidated that miR-577 acts as a tumor inhibitor in many cancers, such as colorectal cancer [11], osteosarcoma [12], and nasopharyngeal carcinoma [13]. Besides, miR-577 impeded cell proliferation and invasion in NSCLC via sponging homeobox A1 [14]. Nevertheless, the relationship between DSCAM-AS1 and miR-577 in NSCLC development has not been clarified. 
Table 1. The clinicopathological parameters of non-small cell lung cancer patients.

\begin{tabular}{ll}
\hline Pathology character & $\mathbf{n}$ \\
\hline Gender & \\
Male & 20 \\
$\quad$ Female & 12 \\
Age, years & \\
$\quad<60$ & 14 \\
$\geq 60$ & 18 \\
Tumor size, cm & \\
$\quad<5$ & 19 \\
$\geq 5$ & 13 \\
TNM stage & \\
$\quad<$ III stage & 20 \\
$\quad \geq$ III stage & 12 \\
Lymph node metastasis & \\
$\quad$ Yes & 22 \\
No & 10 \\
\hline
\end{tabular}

High mobility group box 1 (HMGB1) mainly binds to nucleus DNA and participates in inflammation, tumor growth, and metastasis [15]. More importantly, HMGB1 is closely associated with the development of lung cancer and has been identified as a biomarker of its pathogenesis [16, 17]. For example, HMGB1 upregulation could facilitate cell migration and invasion via the HMGB1-mediated NF- $\kappa \mathrm{B}$ pathway in NSCLC [18].

Hence, we measured the expression of DSCAM-AS1, miR-577, and HMGB1 in NSCLC tissues and cells. Besides, we explored the potential mechanism of DSCAM-AS1 in NSCLC progression.

\section{Patients and methods}

Specimen collection. Thirty-two NSCLC samples and adjacent normal tissues were obtained from thirtytwo patients diagnosed with NSCLC at Huangshi Central Hospital (Affiliated Hospital of Hubei Polytechnic University). The clinicopathological parameters of NSCLC patients are summarized in Table 1 . This study was authorized by the Ethics Committee of Huangshi Central Hospital (Affiliated Hospital of Hubei Polytechnic University). All participants signed written informed consents. Samples were immediately frozen in liquid nitrogen and stored at $-80^{\circ} \mathrm{C}$.

Cell culture. Three NSCLC cell lines (A549, H1299, and H460) and human lung epithelial cells BEAS-2B were obtained from American Type Culture Collection (ATCC, Manassas, VA, USA). PC-9 cell line was purchased from Huzheng (Shanghai, China). Cells were incubated at $37^{\circ} \mathrm{C}$ in Dulbecco's Modified Eagle Medium (DMEM; Solarbio, Shanghai, China) supplemented with $10 \%$ fetal bovine serum (FBS; Solarbio).

Cell transfection. The vectors and oligonucleotides were transfected into NSCLC cells at 70\% confluence using
Lipofectamine 2000 (Invitrogen, Carlsbad, CA, USA). Small interfering RNA (siRNA) against DSCAM-AS1 (si-DSCAMAS1), the negative control siRNA (si-NC), DSCAM-AS1 overexpression vector (DSCAM-AS1), the empty overexpression vector ( $\mathrm{pcDNA}), \mathrm{HMGB} 1$ overexpression vector (HMGB1), miR-577 mimics, the mimics control (mimicsNC), inhibitor miR-577, and the inhibitor control (inhibitor NC) were synthesized by Ribobio (Guangzhou, China).

Lentivirus infection. Lentivirus vectors containing shRNA against DSCAM-AS1 (sh-DSCAM-AS1) or the negative control (sh-NC) were constructed in Genechem (Shanghai, China). $1 \times 10^{6} \mathrm{TU} / \mathrm{ml}$ lentivirus with Polybrene was infected into $\mathrm{H} 460$ cells at $70 \%$ confluence. Subsequently, transfected cells were selected with puromycin.

Quantitative real-time PCR (qRT-PCR). RNA extraction was carried out using Trizol reagent (Invitrogen). Next, RNA was reversely transcribed to cDNA by using the FastQuant RT Kit (Tiangen, Beijing, China). Then, the expression was monitored by SYBR Green PCR Master Mix (Solarbio). GAPDH or U6 was used as an internal control. The primer sequences were used as follows: DSCAM-AS1-F: 5'-CCTATCCCTTTCTCTAAGAA-3', DSCAM-AS1-R， 5'-ACTTCTGCAAAAACGTGCTG-3'; miR-577-F: 5'-TGCGGTAGATAAAATATTGG-3', miR-577-R, 5'-GTGCAGGGTCCGAGGT-3'; HMGB1-F; 5' - TATGGCAAAAGCGGACAAGG-3', HMGB1-R, 5'-CTTCGCAACATCACCAATGGA-3'; GAPDH-F: 5'-GGAGCGAGATCCCTCCAAAAT-3', GAPDH-R, 5'-GGCTGTTGTCATACTTCTCATGG-3'; U6-F: 5'-CTCGCTTCGGCAGCACA-3', U6-R, 5'-AACGCTTCACGAATTTGCGT-3'.

Western blot assay. After extracting the proteins using RIPA buffer (Solarbio), proteins were separated by SDS-PAGE and transferred to polyvinylidene fluoride membranes (Millipore, Billerica, MA, USA). After blocking with 5\% skim milk, the membranes were incubated with primary antibodies overnight at $4^{\circ} \mathrm{C}$ and then incubated with the corresponding secondary antibody (Abcam) for $2 \mathrm{~h}$ at $37^{\circ} \mathrm{C}$. Finally, the signal intensity was detected by the enhanced chemiluminescence system (Thermo Fisher Scientific). The primary antibodies were from Abcam as follows: HMGB1

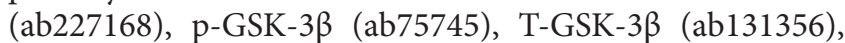
$\beta$-catenin (ab16051), c-Myc (ab39688), CyclinD1 (ab226977), and GAPDH (ab9485).

Cell Counting Kit-8 (CCK-8) assay. Cell viability was evaluated by CCK-8 solution (Dojindo, Kumamoto, Japan). The cells $\left(3.0 \times 10^{3}\right)$ were seeded into a 96-well plate. After incubation for $24 \mathrm{~h}, 48 \mathrm{~h}$, and $72 \mathrm{~h}, \mathrm{CCK}-8$ solution was added to each well. The OD value was measured at $450 \mathrm{~nm}$ with a microplate reader (Bio-Rad, Hercules, CA, USA) after incubation for $2 \mathrm{~h}$.

Flow cytometry. Cell apoptosis was assessed using an Annexin V-FITC/propidium iodide (PI) Apoptosis Detection kit (Invitrogen). Briefly, cells were seeded into a six-well plate and cleaned twice with PBS. Then, cells were stained 
with Annexin V-FITC and PI for $15 \mathrm{~min}$. At last, the apoptotic rate was monitored by Attune NxT Flow Cytometer (Thermo Fisher Scientific).

Transwell assay. For cell migration, cells were placed in the upper chamber with a serum-free medium. Besides, $10 \%$ FBS was added into the lower chamber. The migrated cells were treated with methanol and stained with crystal violet for $20 \mathrm{~min}$ after incubation for $24 \mathrm{~h}$. Three fields were randomly selected and counted under a microscope. For cell invasion, transwell chambers were coated with Matrigel (BD Biosciences, San Diego, CA, USA), and the other experimental procedures were unchanged.

Dual-luciferase reporter assay. The sequences of DSCAM-AS1 or HMGB1 3'UTR containing wild-type or mutant binding site of miR-577 were inserted into pGL3 vector (Promega, Madison, WI, USA) to construct WT-DSCAMAS1, MUT-DSCAM-AS1, HMGB1 3'UTR-WT or HMGB1 3'UTR-MUT, respectively. Then, the corresponding vector and miR-577 mimics or mimics-NC were co-transfected into cells. The luciferase activity was detected using Dual-Luciferase Reporter Kit (Promega).

Xenograft experiment. Male $\mathrm{BALB} / \mathrm{c}$ nude mice (6 weeks) were randomly divided into two groups ( $n=5 /$ group).
H460 cells were infected with lentivirus harboring sh-NC or sh-DSCAM-AS1, respectively. Then, cells $\left(5 \times 10^{6}\right)$ were injected subcutaneously into the right back of nude mice. Tumor volume was measured every 7 days. After mice were sacrificed, the xenograft was removed and weighed. The levels of DSCAM-AS1, miR-577, and HMGB1 were detected by qRT-PCR. The experiment was approved by the Animal Welfare Committee of Huangshi Central Hospital (Affiliated Hospital of Hubei Polytechnic University).

Statistical analysis. At least three independent experiments were performed, and the data were shown as mean \pm standard deviation. Differences were assessed using Student's t-test or one-way analysis of variance. GraphPad Prism 7.0 software (GraphPad, San Diego, CA, USA) was executed to analyze data. A p-value $<0.05$ was considered to be statistically significant.

\section{Results}

DSCAM-AS1 and HMGB1 were upregulated in NSCLC tissues and cells. Firstly, DSCAM-AS1 expression was overtly enhanced in NSCLC tissues compared with normal tissues (Figure 1A). Consistently, DSCAM-AS1 expression was
A

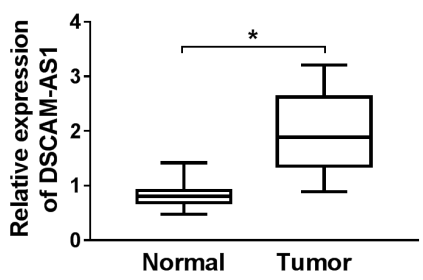

D

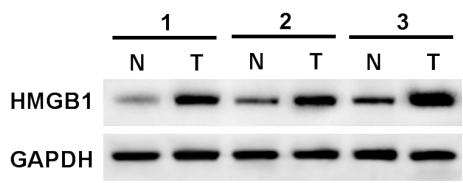

$\mathbf{F}$

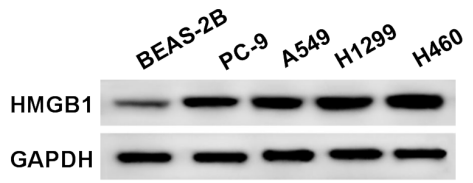

B
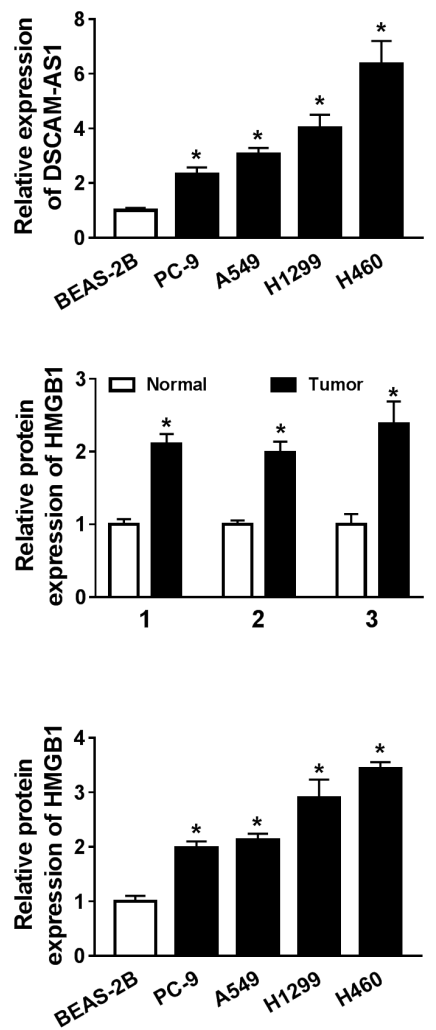

C

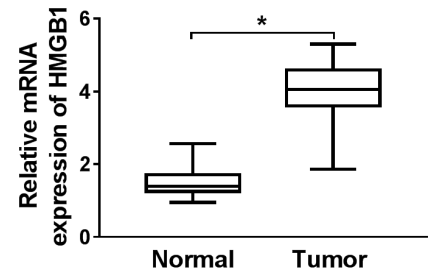

E

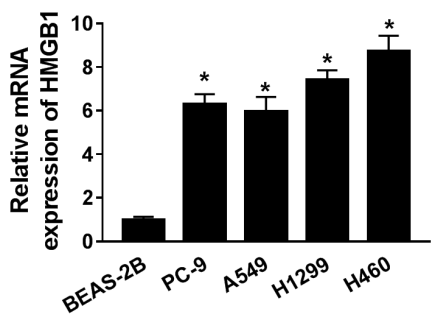

G

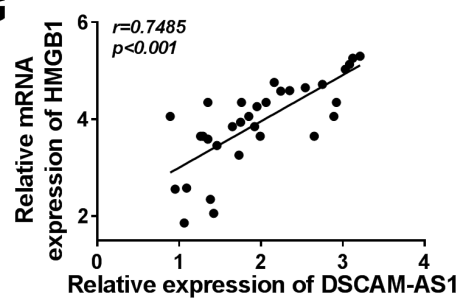

Figure 1. DSCAM-AS1 and HMGB1 were upregulated in NSCLC tissues and cells. A) The expression of DSCAM-AS1 was measured in NSCLC tissues and adjacent normal tissues by qRT-PCR. B) DSCAM-AS1 expression was detected in BEAS-2B cells and four NSCLC cell lines by qRT-PCR. C-F) The mRNA and protein levels of HMGB1 were examined in NSCLC tissues and cells by qRT-PCR or western blot assay. G) The correlation between DSCAM-AS1 and HMGB1 expression was shown in NSCLC tissues. ${ }^{*} \mathbf{p}<0.05$ 
prominently higher in NSCLC cells (PC-9, A549, H1299, and $\mathrm{H} 460$ ) than that in BEAS-2B cells (Figure 1B). Moreover, the mRNA and protein levels of HMGB1 were obviously elevated in NSCLC tissues and cells (Figures 1C-1F). DSCAM-AS1 expression was positively correlated with HMGB1 mRNA (Figure 1G). These data implied that DSCAM-AS1 and HMGB1 might play a vital role in NSCLC progression.
DSCAM-AS1 induced cell proliferation, migration and invasion, and restrained cell apoptosis in NSCLC cells. To investigate the effect of DSCAM-AS1 on NSCLC progression, H1299 and $\mathrm{H} 460$ cells were transfected with si-NC, si-DSCAM-AS1, pcDNA, or DSCAM-AS1, respectively. The results of qRT-PCR showed that DSCAM-AS1 knockdown effectively inhibited DSCAM-AS1 expression,
A

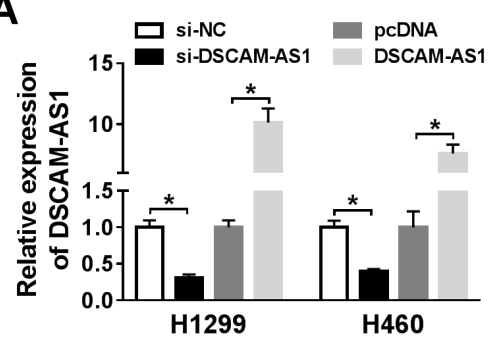

B

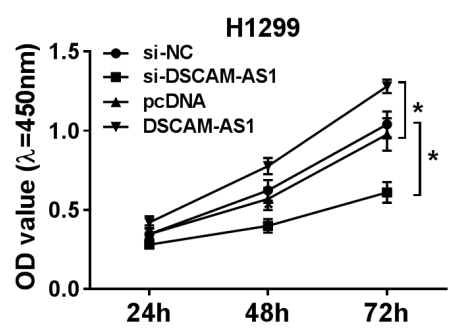

D

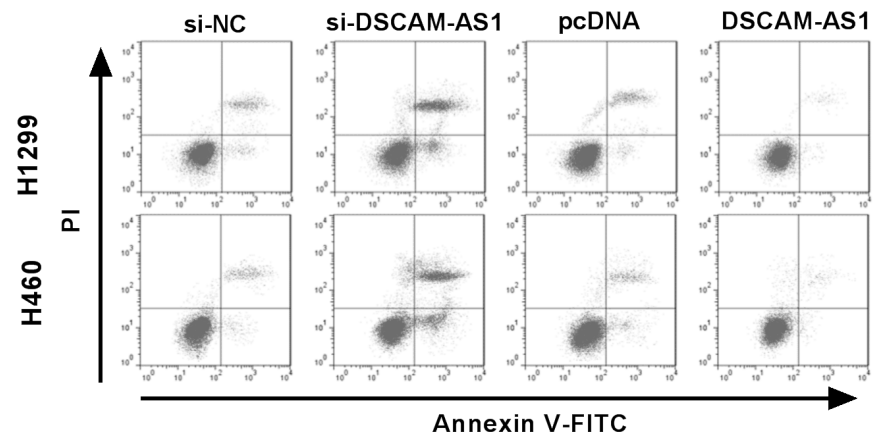

E

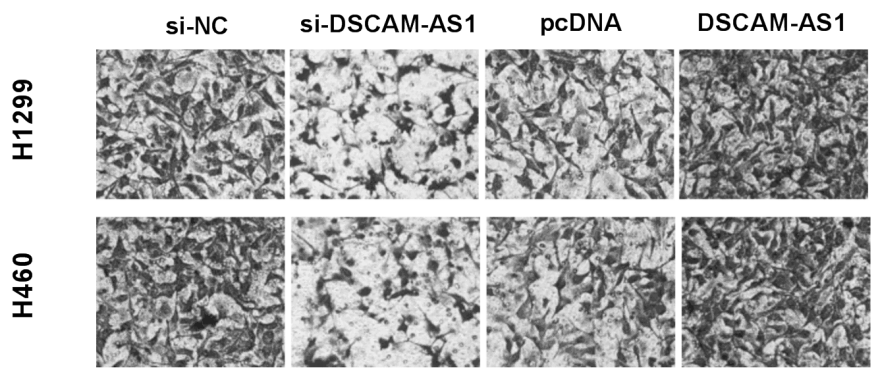

$\mathbf{F}$
C
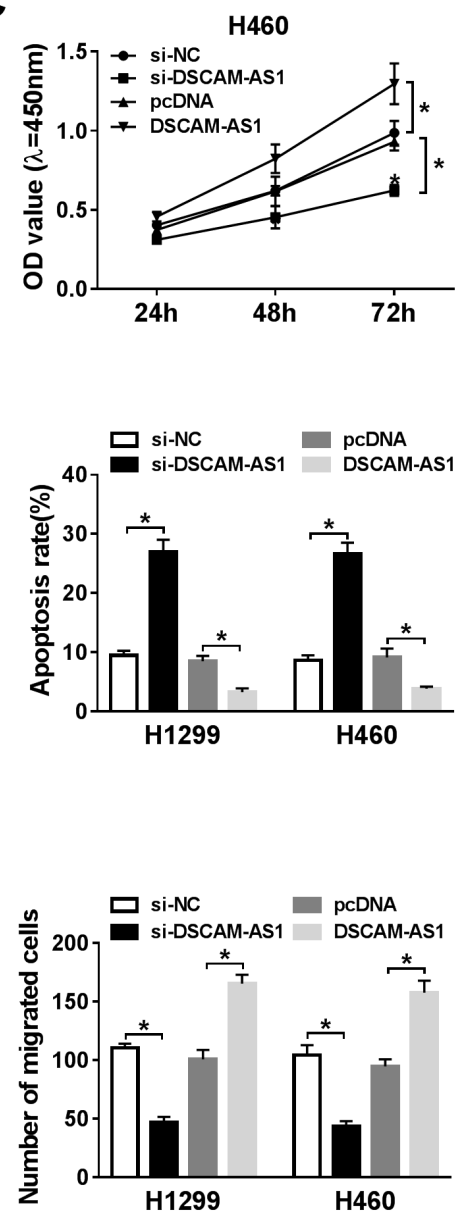

Figure 2. DSCAM-AS1 induced cell proliferation, migration and invasion, and restrained cell apoptosis in NSCLC cells. A-F) H1299 and H460 cells were transfected with si-NC, si-DSCAM-AS1, pcDNA, or DSCAM-AS1, respectively. A) DSCAM-AS1 expression was measured in transfected cells using qRT-PCR. B, C) CCK-8 assay was used to assess cell viability. D) Cell apoptosis rate was evaluated by flow cytometry. E, F) Cell migration and invasion were detected by transwell assay. ${ }^{\star} \mathbf{p}<0.05$ 
and DSCAM-AS1 overexpression dramatically enhanced DSCAM-AS1 expression (Figure 2A). CCK-8 assay exhibited that DSCAM-AS1 knockdown especially suppressed cell viability, and DSCAM-AS1 overexpression markedly triggered cell viability (Figures 2B, 2C). Flow cytometry assay suggested that the cell apoptosis rate was conspicuously increased by inhibiting DSCAM-AS1, and overtly reduced by upregulating DSCAM-AS1 (Figure 2D). In addition, transwell assay revealed that cell migration and invasion were apparently hindered after transfection with si-DSCAMAS1, and notably facilitated after transfection with DSCAMAS1 (Figures 2E, 2F). All these data indicated that DSCAMAS1 induced cell proliferation, migration and invasion, and restrained cell apoptosis in NSCLC cells.

HMGB1 overexpression reversed the effects of DSCAM-AS1 knockdown on NSCLC progression. First of all, the mRNA and protein levels of HMGB1 were obviously decreased in H1299 and H460 cells transfected with
si-DSCAM-AS1 compared with the si-NC group, and greatly elevated in DSCAM-AS1 group compared with pcDNA group (Figures 3A, 3B). Subsequently, to explore the role of HMGB1 in NSCLC progression, H1299 and H460 cells were transfected with si-NC, si-DSCAM-AS1, si-DSCAMAS1+pcDNA, or si-DSCAM-AS1+HMGB1, respectively. CCK-8 assay showed that DSCAM-AS1 knockdown significantly restrained cell viability, while overexpression of HMGB1 attenuated the effect (Figures 3C, 3D). DSCAMAS1 knockdown substantially contributed to cell apoptosis, whereas the effect was rescued after transfection with HMGB1 (Figure 3E). Furthermore, DSCAM-AS1 silencing remarkably blocked cell migration and invasion, but the effect was relieved by upregulating HMGB1 (Figures 3F, 3G). These data reflected that DSCAM-AS1 knockdown might suppress NSCLC progression by modulating HMGB1.

DSCAM-AS1 targeted miR-577 to regulate HMGB1 expression. Online database starBase 3.0 predicted that
A

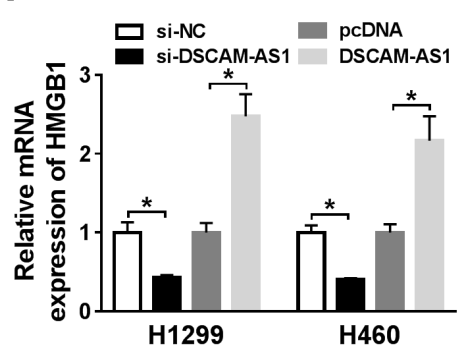

C

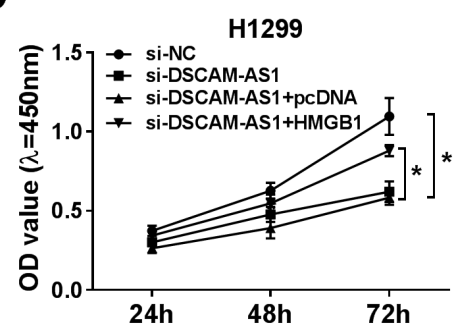

$\mathbf{F}$

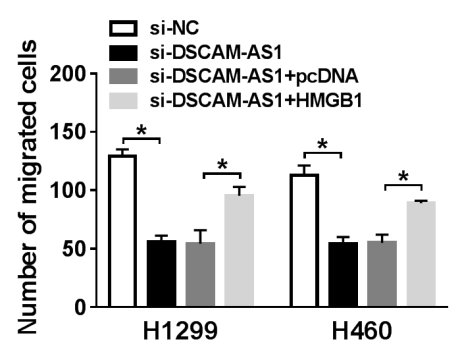

B

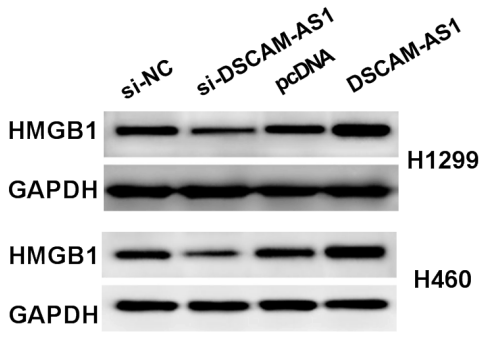

D

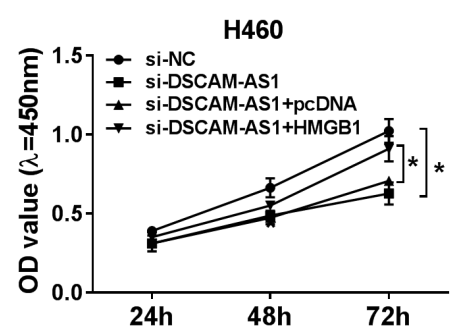

G

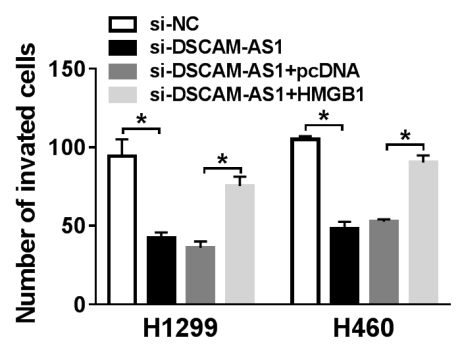

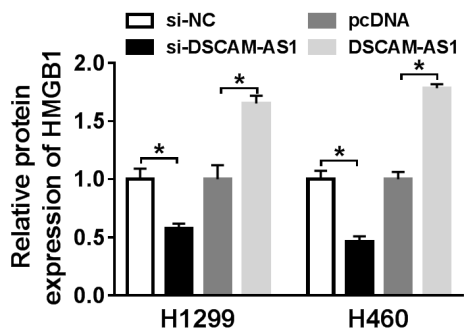

E

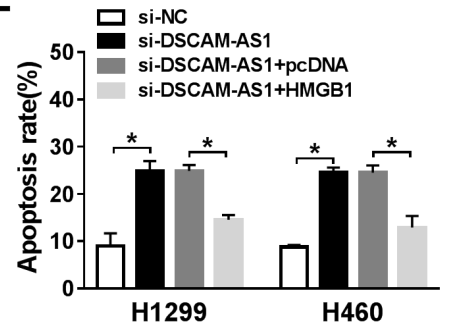

Figure 3. HMGB1 overexpression reversed the effects of DSCAM-AS1 knockdown on NSCLC progression. A, B) The mRNA and protein levels of HMGB1 were measured in $\mathrm{H} 1299$ and $\mathrm{H} 460$ cells transfected with si-NC, si-DSCAM-AS1, pcDNA, or DSCAM-AS1 by using qRT-PCR or western blot assay. C-G) H1299 and H460 cells were transfected with si-NC, si-DSCAM-AS1, si-DSCAM-AS1+pcDNA, or si-DSCAM-AS1+HMGB1, respectively. C, D) Cell viability was assessed by CCK-8 assay. E) Flow cytometry was conducted to monitor the cell apoptotic rate. F, G) Transwell assay was utilized to detect cell migration and invasion. ${ }^{*} \mathrm{p}<0.05$ 
DSCAM-AS1 and miR-577 have putative binding sites (Figure 4A). To verify whether DSCAM-AS1 binds to miR-577, dual-luciferase reporter assay was applied, and the results suggested that miR-577 mimics notably reduced the luciferase activity of WT-DSCAM-AS1, while luciferase activity of MUT-DSCAM-AS1 was not affected in both H1299 and $\mathrm{H} 460$ cells (Figures 4B, 4C). In addition, the expression of miR-577 was detected in $\mathrm{H} 1299$ and $\mathrm{H} 460$ cells transfected with si-NC, si-DSCAM-AS1, pcDNA, or DSCAM-AS1, respectively. The results indicated that DSCAM-AS1 knockdown obviously elevated miR-577 expression, while DSCAMAS1 overexpression evidently reduced miR-577 expression in H1299 and H460 cells (Figure 4D). Next, starBase 3.0 predicted that miR-577 and HMGB1 have binding sites (Figure 4E). Dual-luciferase reporter assay revealed that mature miR-577 strikingly decreased the luciferase activity of HMGB1 3'UTR-WT, whereas not affected the luciferase activity of HMGB1 3'UTR-MUT in H1299 and H460 cells (Figures 4F, 4G). Additionally, the expression of HMGB1 was measured in $\mathrm{H} 1299$ and $\mathrm{H} 460$ cells transfected with mimics-NC, miR-577 mimics, miR-577 mimics+pcDNA or miR-577 mimics+DSCAM-AS1. The results showed that the mRNA and protein levels of HMGB1 were distinctly reduced in the miR-577 mimics group compared to the mimics-NC group, while HMGB1 expression was reversed after transfection with DSCAM-AS1 (Figures 4H, 4I). Besides, miR-577 expression was dramatically inhibited in NSCLC tissues and cells (Figures 4J,4K), and negatively correlated with the expression of DSCAM-AS1 and HMGB1 in NSCLC tissues (Figures 4L, 4M). Taken together, these data demonstrated that DSCAM-AS1 regulated HMGB1 expression via sponging $\mathrm{miR}-577$.

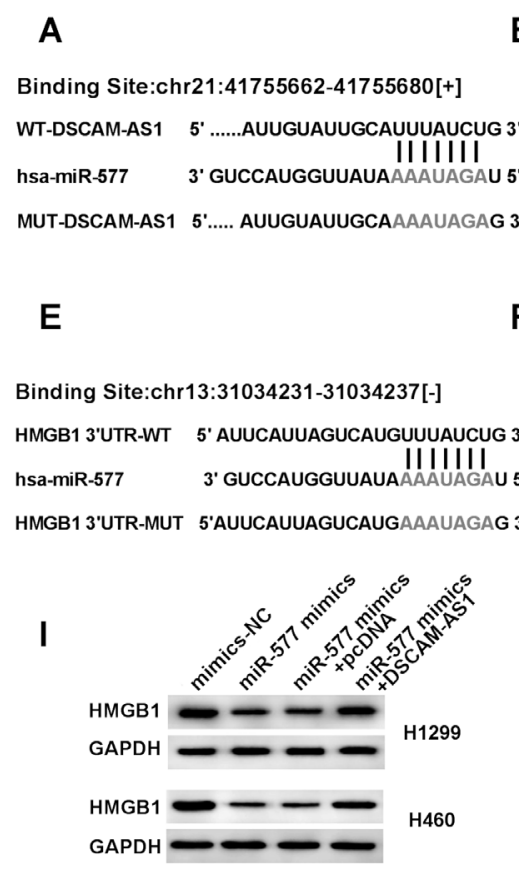

$\mathbf{L}$

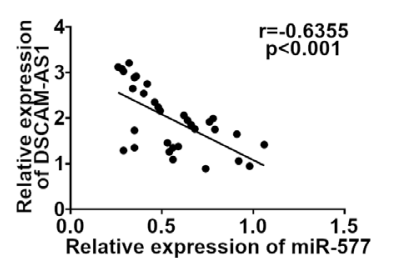

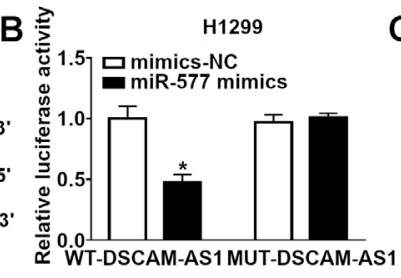
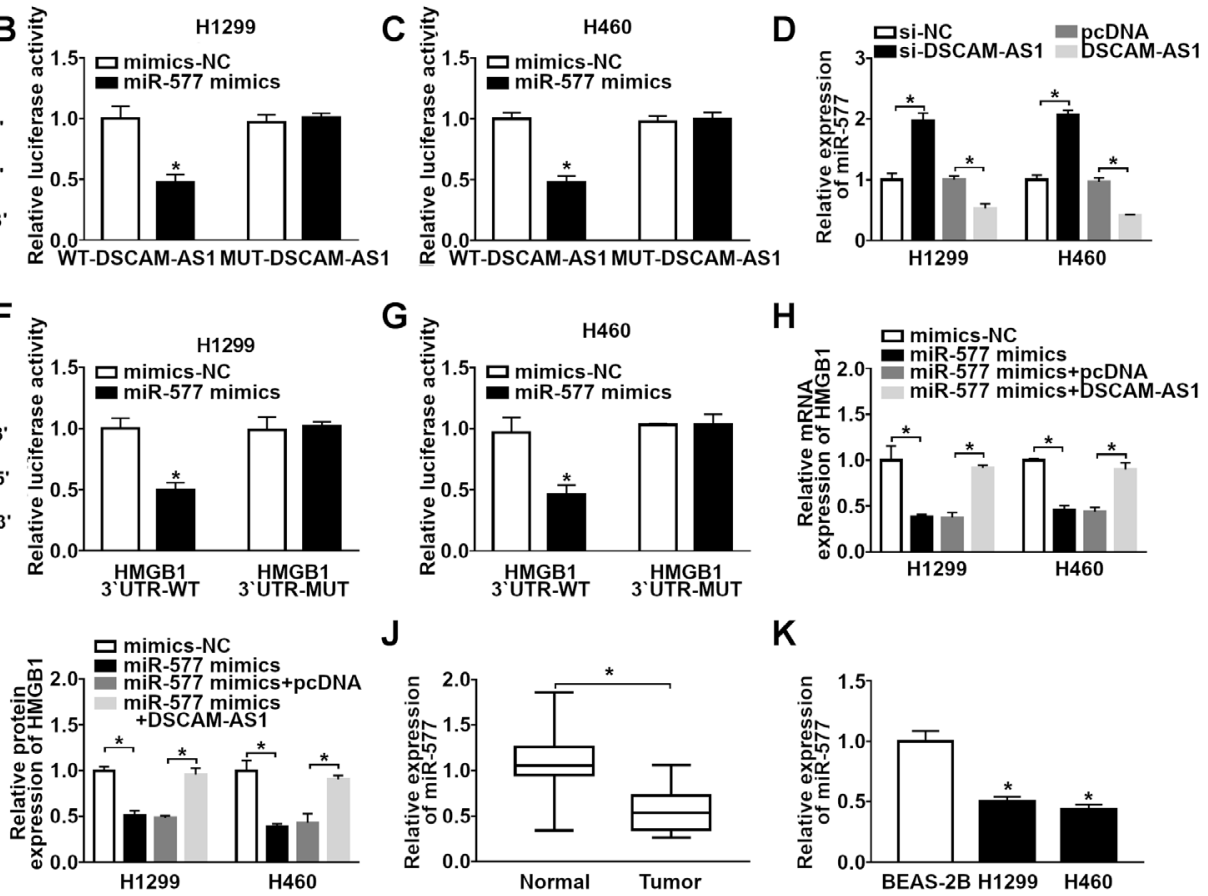

\section{J}
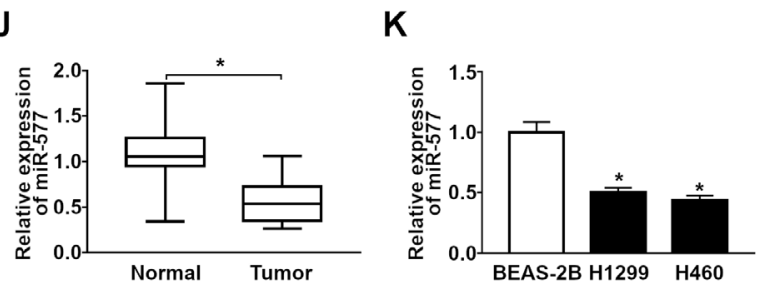

Figure 4. DSCAM-AS1 targeted miR-577 to regulate HMGB1 expression. A) The putative binding sites of DSCAM-AS1 and miR-577 were suggested. B, C) Dual-luciferase reporter assay was performed to analyze the luciferase activity of H1299 and H460 cells co-transfected with WT-DSCAM-AS1 or MUT-DSCAM-AS1 and miR-577 mimics or mimics-NC. D) The level of miR-577 was detected in H1299 and H460 cells transfected with si-NC, si-DSCAM-AS1, pcDNA, or DSCAM-AS1, respectively. E) The predicted binding sites of miR-577 and HMGB1 were exhibited. F, G) The luciferase activity was analyzed in H1299 and H460 cells transfected with HMGB1 3'UTR-WT or HMGB1 3'UTR-MUT and miR-577 mimics or mimics-NC by dual-luciferase reporter assay. H, I) Expression of HMGB1 was detected in $\mathrm{H} 1299$ and $\mathrm{H} 460$ cells transfected with mimics-NC, miR-577 mimics, miR577 mimics+pcDNA or miR-577 mimics+DSCAM-AS1. J, K) MiR-577 expression was measured in NSCLC tissues and cells by qRT-PCR. L, M) The correlation between miR-577 and DSCAM-AS1 or HMGB1 was shown. ${ }^{*} \mathbf{p}<0.05$ 

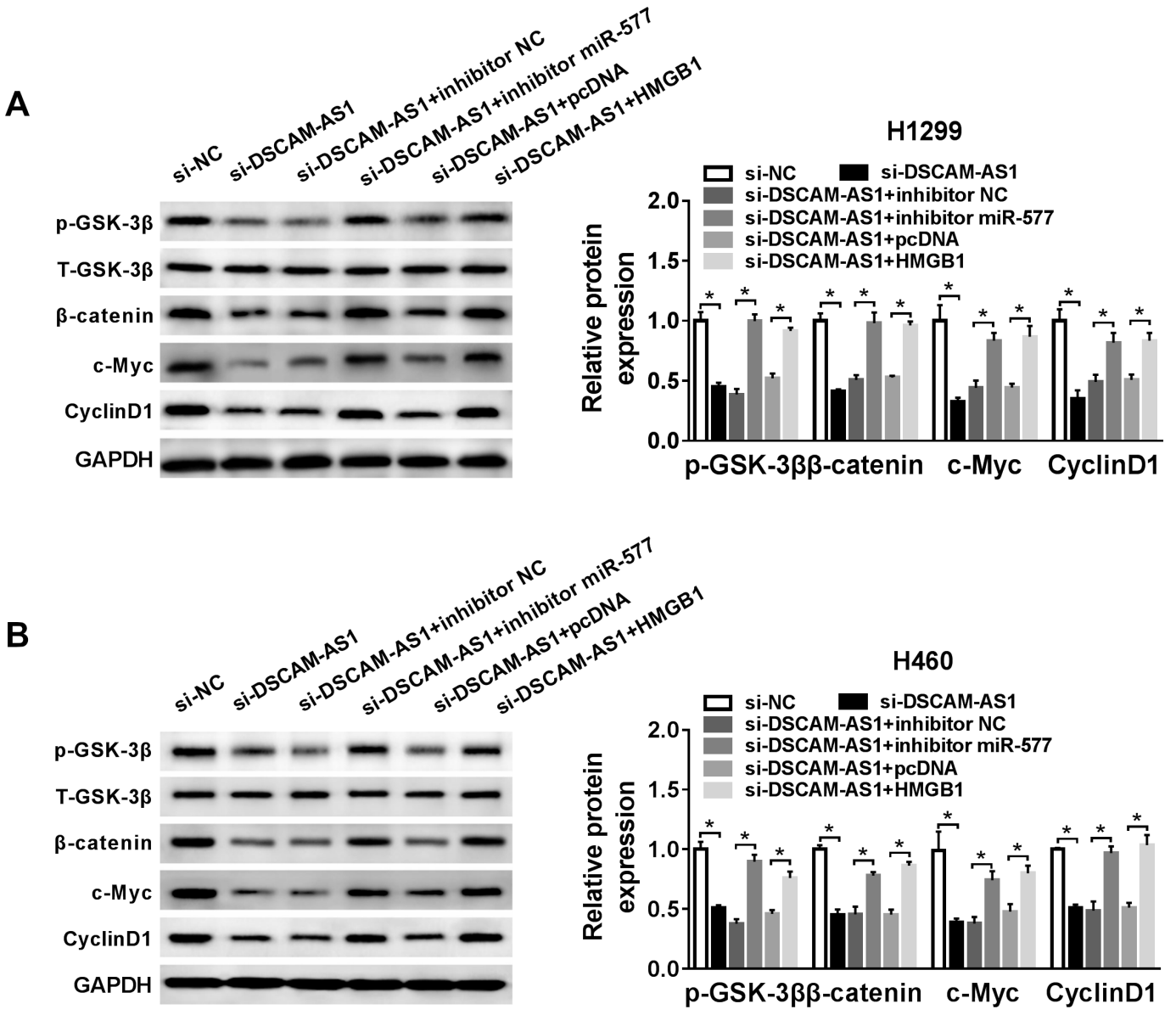

Figure 5. DSCAM-AS1 regulated Wnt/ $\beta$-catenin pathway by affecting miR-577 and HMGB1. A, B) The protein levels of p-GSK-3 $\beta$, $\beta$-catenin, c-Myc, and Cyclin D1 were tested in $\mathrm{H} 1299$ and $\mathrm{H} 460$ cells transfected with si-NC, si-DSCAM-AS1, si-DSCAM-AS1+inhibitor NC, si-DSCAM-AS1+inhibitor miR-577, si-DSCAM-AS1+pcDNA, or si-DSCAM-AS1+HMGB1 using western blot assay. ${ }^{*} \mathbf{p}<0.05$

DSCAM-AS1 regulated the Wnt/ $\beta$-catenin pathway by affecting miR-577 and HMGB1. To explore the effect of DSCAM-AS1 on the Wnt/ $\beta$-catenin pathway, H1299 and H460 cells were transfected with si-NC, si-DSCAM-AS1, si-DSCAM-AS1+inhibitor NC, si-DSCAM-AS1+inhibitor miR-577, si-DSCAM-AS1+pcDNA, or si-DSCAM-AS1+ HMGB1, respectively. Western blot analysis exhibited that interference of DSCAM-AS1 remarkably inhibited the expression of p-GSK-3 $\beta, \beta$-catenin, c-Myc, and Cyclin D1 in H1299 and H460 cells, while inhibitor miR-577 or HMGB1 overexpression could restore the effect (Figures 5A, 5B). These data disclosed that DSCAM-AS1 could regulate the Wnt $/ \beta$ catenin pathway by modulating miR-577 and HMGB1.

The knockdown of DSCAM-AS1 hindered tumor growth in vivo. To further determine the effect of DSCAMAS1 on NSCLC tumorigenesis, H460 cells transfected with sh-NC or sh-DSCAM-AS1 were injected subcutaneously into nude mice. Tumor volume and weight in the sh-DSCAM-AS1 group were conspicuously reduced compared with negative control (Figures 6A, 6B). In addition, the expression levels of DSCAM-AS1and HMGB1 were apparently lower, while miR-577 expression was notably higher in the sh-DSCAMAS1 group than that in the sh-NC group (Figure 6C). These data manifested that the DSCAM-AS1 knockdown suppressed the tumor growth of NSCLC in vivo.

\section{Discussion}

Recently, DSCAM-AS1 has been reported as an oncogene in several cancers [19]. DSCAM-AS1 contributed to breast cancer progression via regulating miR-204-5p/RRM2 axis [20]. In ovarian cancer, DSCAM-AS1 induced tumor development and was associated with poor prognosis by increasing SOX4 expression [21]. Furthermore, DSCAMAS1 was overtly elevated in melanoma and facilitated melanoma progression via targeting miR-136 [22]. Similar to previous studies, the level of DSCAM-AS1 was distinctly increased in NSCLC tissues and cell lines. Also, the upregulation of DSCAM-AS1 induced cell proliferation, migration and invasion, and restrained cell apoptosis in NSCLC. 
A

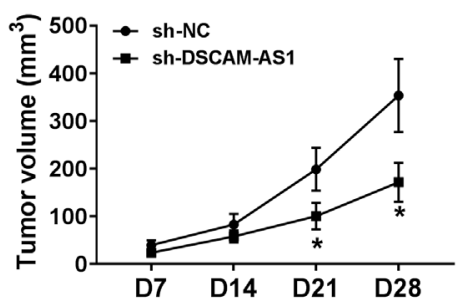

B

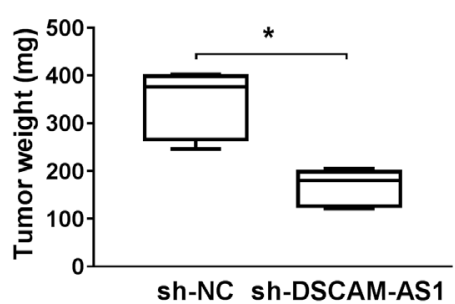

C

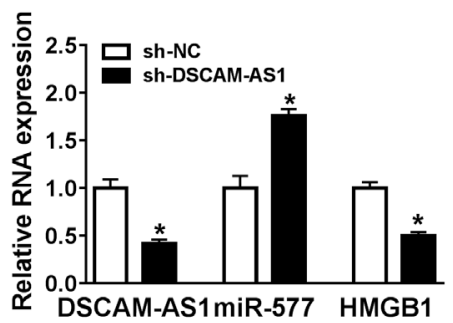

Figure 6. The knockdown of DSCAM-AS1 hindered tumor growth in vivo. A-C) H460 cells transfected with sh-NC or sh-DSCAM-AS1 were subcutaneously injected into nude mice. A) Tumor volume was measured every 7 days. B) Tumor weight was examined after mice were sacrificed. C) The expression levels of DSCAM-AS1, miR-577, and HMGB1 were detected by qRT-PCR. * $<<0.05$

More and more evidence suggested that lncRNAs could regulate target genes by acting as competing endogenous RNAs for miRNAs [23]. Based on this, we predicted the putative targets of DSCAM-AS1 by starBase3.0 online database and selected miR-577 as the research target according to the previous studies. miR-577 was a tumor suppressor in a variety of cancers [24]. However, Luo et al. found that miR-577 was a tumor promotor and modulated TGF- $\beta$-induced progression and metastasis of gastric cancer [25]. Yin et al. showed that miR-577 restricted epithelialmesenchymal transition and invasion in breast cancer via regulating Rab25 [26]. Xue et al. found that miR-577 suppressed the development of papillary thyroid carcinoma via targeting sphingosine kinase 2 (SphK2) [27]. Another previous research exhibited that miR-577 hindered colorectal cancer progression by upregulating HSP27 expression [28]. In NSCLC, miR-577 inhibited tumor growth via WNT2B mediated Wnt/ $\beta$-catenin pathway [29]. Consistent with the previous study, miR-577 was remarkably downregulated in NSCLC. Besides, HMGB1 overexpression reversed the effect on NSCLC progression induced by DSCAM-AS1 knockdown.

Increasing evidence suggested that the $W n t / \beta$-catenin pathway participates in the pathogenesis of multiple tumors [30]. Studies have shown that tumor metastasis is mainly due to $\mathrm{Wnt} / \beta$-catenin regulating the transcription of EMT-related genes [31,32]. Wang et al. found that miR-665 suppressed the development of retinoblastoma via elevating HMGB1 expression and inhibiting the Wnt/ $\beta$ catenin pathway [33]. HMGB1 could modulate chronic renal disease-induced vascular calcification by regulating the Wnt $/ \beta$-catenin pathway [34]. HMGB1 triggered NSCLC cell migration by activating av $\beta 3 /$ FAK through the TLR4/NF- $\kappa B$ signaling pathway [35]. In this study, HMGB1 was a target of miR-577. DSCAM-AS1 enhanced HMGB1 expression by sponging miR-577 and modulated Wnt/ $\beta$-catenin pathway via regulating miR-577 and HMGB1.

In this study, DSCAM-AS1 was highly expressed in NSCLC. DSCAM-AS1 might facilitate NSCLC progression via regulating the miR-577/HMGB1 axis and activating $\mathrm{Wnt} / \beta$-catenin pathway.

\section{References}

[1] HIRSCH FR, SCAGLIOTTI GV, MULSHINE JL, KWON R, CURRAN WJ JR et al. Lung cancer: current therapies and new targeted treatments. Lancet 2017; 389: 299-311. https:// doi.org/10.1016/S0140-6736(16)30958-8

[2] SEIJO LM, PELED N, AJONA D, BOERI M, FIELD JK et al. Biomarkers in lung cancer screening: achievements, promises, and challenges. J Thorac Oncol 2019; 14: 343-357. https:// doi.org/10.1016/j.jtho.2018.11.023

[3] TESTA U, CASTELLI G, PELOSI E. Lung cancers: molecular characterization, clonal heterogeneity and evolution, and cancer stem cells. Cancers (Basel) 2018; 10: E248. https://doi. org/10.3390/cancers 10080248

[4] ZHANG L, DONG Y, WANG Y, GAO J, LV J et al. Et al. Long non-coding RNAs in ocular diseases: new and potential therapeutic targets. FEBS J 2019; 286: 2261-2272. https:// doi.org/10.1111/febs.14827

[5] CHEN Y, BAO C, ZHANG X, LIN X, HUANG H et al. Long non-coding RNA HCG11 modulates glioma progression through cooperating with miR-496/CPEB3 axis. Cell Prolif 2019; 52: e12615. https://doi.org/10.1111/cpr.12615

[6] ZHANG S, ZHANG X, SUN Q, ZHUANG C, LI G et al. LncRNA NR2F2-AS1 promotes tumourigenesis through modulating BMI1 expression by targeting miR-320b in nonsmall cell lung cancer. J Cell Mol Med 2019; 23: 2001-2011. https://doi.org/10.1111/jcmm.14102

[7] WANG J, CAI H, DAI Z, WANG G. Down-regulation of lncRNA XIST inhibits cell proliferation via regulating miR-744/RING1 axis in non-small cell lung cancer. Clin Sci (Lond) 2019; 133: 1567-1579. https://doi.org/10.1042/ CS20190519

[8] SUN W, LI AQ, ZHOU P, JIANG YZ, JIN X et al. DSCAMAS1 regulates the G/S cell cycle transition and is an independent prognostic factor of poor survival in luminal breast cancer patients treated with endocrine therapy. Cancer Med 2018; 7: 6137-6146. https://doi.org/10.1002/ cam4.1603

[9] LIAO J, XIE N. Long noncoding RNA DSCAM-AS1 functions as an oncogene in non-small cell lung cancer by targeting BCL11A. Eur Rev Med Pharmacol Sci 2019; 23: 10871092. https://doi.org/10.26355/eurrev_201902_16998 
[10] ONG SG, LEE WH, KODO K, WU JC. MicroRNA-mediated regulation of differentiation and trans-differentiation in stem cells. Adv Drug Deliv Rev 2015; 88: 3-15. https://doi. org/10.1016/j.addr.2015.04.004

[11] WANG Y, LU Z, WANG N, FENG J, ZHANG J et al. Long noncoding RNA DANCR promotes colorectal cancer proliferation and metastasis via miR-577 sponging. Exp Mol Med. 2018;50(5):1-17.https://doi.org/10.1038/s12276-018-0082-5

[12] JIANG Z, JIANG C, FANG J. Up-regulated lnc-SNHG1 contributes to osteosarcoma progression through sequestration of miR-577 and activation of WNT2B/Wnt/ $\beta$-catenin pathway. Biochem Biophys Res Commun 2018; 495: 238-245. https://doi.org/10.1016/j.bbrc.2017.11.012

[13] WEI H, LIU D, SUN J, MAO Y, ZHAO L et al. Circular RNA circ_0008450 upregulates CXCL9 expression by targeting miR-577 to regulate cell proliferation and invasion in nasopharyngeal carcinoma. Exp Mol Pathol 2019; 110: 104288. https://doi.org/10.1016/j.yexmp.2019.104288

[14] MEN L, NIE D, NIE H. microRNA-577 inhibits cell proliferation and invasion in non-small cell lung cancer by directly targeting homeobox A1. Mol Med Rep 2019; 19: 1875-1882. https://doi.org/10.3892/mmr.2019.9804

[15] PAUDEL YN, ANGELOPOULOU E, PIPERI C, BALASUBRAMANIAM VRMT, OTHMAN I et al. Enlightening the role of high mobility group box 1 (HMGB1) in inflammation: Updates on receptor signalling. Eur J Pharmacol 2019; 858: 172487. https://doi.org/10.1016/j.ejphar.2019.172487

[16] WU XJ, CHEN YY, GONG CC, PEI DS. The role of highmobility group protein box 1 in lung cancer. J Cell Biochem 2018; 119: 6354-6365. https://doi.org/10.1002/jcb.26837

[17] WANG M, GAUTHIER A, DALEY L, DIAL K, WU J et al. The Role of HMGB1, a nuclear damage-associated molecular pattern molecule, in the pathogenesis of lung diseases. Antioxidants and Redox Signaling 2019; 31: 954-993. https://doi. org/10.1089/ars.2019.7818

[18] WU D, LIU J, CHEN J, HE H, MA H et al. miR-449a suppresses tumor growth, migration, and invasion in non-small cell lung cancer by targeting a HMGB1-mediated NF- $\mathrm{kB}$ signaling pathway. Oncol Res 2019; 27: 227-235. https://doi.org /10.3727/096504018X15213089759999

[19] XU S, KONG D, CHEN Q, PING Y, PANG D. Oncogenic long noncoding RNA landscape in breast cancer. Mol Cancer 2017; 16: 129. https://doi.org/10.1186/s12943-017-0696-6

[20] LIANG WH, LI N, YUAN ZQ, QIAN XL et al. . DSCAMAS1 promotes tumor growth of breast cancer by reducing miR-204-5p and up-regulating RRM2. Mol Carcinog 2019; 58: 461-473. https://doi.org/10.1002/mc.22941

[21] LI Y, HAO J, JIANG YM, LIU Y et al. Long non-coding RNA DSCAM-AS1 indicates a poor prognosis and modulates cell proliferation, migration and invasion in ovarian cancer via upregulating SOX4. Eur Rev Med Pharmacol Sci 2019; 23: 4143-4148. https://doi.org/10.26355/eurrev_201905_17916

[22] HUANG YL, XU Q, WANG X. Long noncoding RNA DSCAM-AS1 is associated with poor clinical prognosis and contributes to melanoma development by sponging miR-136. Eur Rev Med Pharmacol Sci 2019; 23: 2888-2897. https://doi.org/10.26355/eurrev_201904_17567
[23] CHAN JJ, TAY Y. Noncoding RNA:RNA regulatory networks in cancer. Int J Mol Sci 2018; 19: E1310. https://doi. org/10.3390/ijms19051310

[24] ZHANG W, SHEN C, LI C, YANG G, LIU H et al. miR577 inhibits glioblastoma tumor growth via the Wnt signaling pathway. Mol Carcinog 2016; 55: 575-585. https://doi. org/10.1002/mc.22304

[25] LUO Y, WU J, WU Q, LI X, WU J et al. miR-577 regulates TGF- $\beta$ induced cancer progression through a SDPR-modulated positive-feedback loop with ERK-NF-kB in gastric cancer. Mol Ther 2019; 27: 1166-1182. https://doi.org/10.1016/j. ymthe.2019.02.002

[26] YIN C, MOU Q, PAN X, ZHANG G, LI H et al. MiR-577 suppresses epithelial-mesenchymal transition and metastasis of breast cancer by targeting Rab25. Thorac Cancer 2018; 9: 472-479. https://doi.org/10.1111/1759-7714.12612

[27] XUE KC, HU DD, ZHAO L, LI N, SHEN HY. MiR-577 inhibits papillary thyroid carcinoma cell proliferation, migration and invasion by targeting SphK2. Eur Rev Med Pharmacol Sci 2017; 21: 3794-3800.

[28] JIANG H, JU H, ZHANG L, LU H, JIE K. microRNA-577 suppresses tumor growth and enhances chemosensitivity in colorectal cancer. J Biochem Mol Toxicol 2017; 31. https:// doi.org/10.1002/jbt. 21888

[29] WANG B, SUN L, LI J, JIANG R. miR-577 suppresses cell proliferation and epithelial-mesenchymal transition by regulating the WNT2B mediated Wnt/ $\beta$-catenin pathway in nonsmall cell lung cancer. Mol Med Rep 2018; 18: 2753-2761. https://doi.org/10.3892/mmr.2018.9279

[30] WANG W, SMITS R, HAO H, HE C. Wnt/ $\beta$-catenin signaling in liver cancers. Cancers (Basel) 2019; 11: E926. https:// doi.org/10.3390/cancers11070926

[31] VU T, DATTA PK. Regulation of EMT in colorectal cancer: a culprit in metastasis. Cancers (Basel) 2017; 9: E171. https:// doi.org/10.3390/cancers9120171

[32] GHAHHARI NM, BABASHAH S. Interplay between microRNAs and $\mathrm{WNT} / \beta$-catenin signalling pathway regulates epithelial-mesenchymal transition in cancer. Eur J Cancer 2015; 51: 1638-1649. https://doi.org/10.1016/j. ejca.2015.04.021

[33] WANG S, DU S, LV Y, ZHANG F, WANG W. MicroRNA-665 inhibits the oncogenicity of retinoblastoma by directly targeting high-mobility group box 1 and inactivating the Wnt/ $\beta$-catenin pathway. Cancer Manag Res 2019; 11: 3111-3123. https://doi.org/10.2147/CMAR.S200566

[34] JIN X, RONG S, YUAN W, GU L, JIA J et al. High mobility group box 1 promotes aortic calcification in chronic kidney disease via the Wnt/ $\beta$-catenin pathway. Front Physiol 2018; 9: 665. https://doi.org/10.3389/fphys.2018.00665

[35] ZHU J, LUO J, LI Y, JIA M, WANG Y et al. HMGB1 induces human non-small cell lung cancer cell motility by activating integrin $\alpha \mathrm{v} \beta 3 / \mathrm{FAK}$ through TLR4/NF- $\mathrm{KB}$ signaling pathway. Biochem Biophys Res Commun 2016; 480: 522-527. https:// doi.org/10.1016/j.bbrc.2016.10.052 